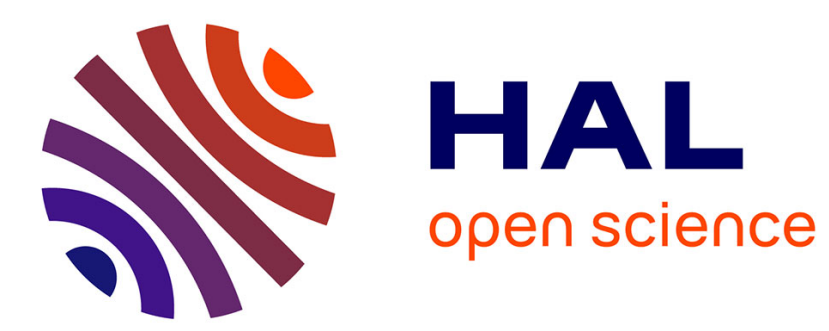

\title{
COMPORTEMENT DYNAMIQUE D'UNE NUANCE DE BERYLLIUM
}

D. Montoya, G. Naulin, J. Ansart

\section{To cite this version:}

D. Montoya, G. Naulin, J. Ansart. COMPORTEMENT DYNAMIQUE D'UNE NUANCE

DE BERYLLIUM. Journal de Physique IV Proceedings, 1991, 01 (C3), pp.C3-27-C3-34. 10.1051/jp4:1991304 . jpa-00249895

\section{HAL Id: jpa-00249895 https://hal.science/jpa-00249895}

Submitted on 1 Jan 1991

HAL is a multi-disciplinary open access archive for the deposit and dissemination of scientific research documents, whether they are published or not. The documents may come from teaching and research institutions in France or abroad, or from public or private research centers.
L'archive ouverte pluridisciplinaire HAL, est destinée au dépôt et à la diffusion de documents scientifiques de niveau recherche, publiés ou non, émanant des établissements d'enseignement et de recherche français ou étrangers, des laboratoires publics ou privés. 


\title{
COMPORTEMENT DYNAMIQUE D'UNE NUANCE DE BERYLLIUM
}

\author{
D. MONTOYA, G. NAULIN et J.P. ANSART
}

Commissariat à I'Énergie Atomique, Centre d'Études de Bruyères le Châtel, BP. 12, F-91680 Bruyères-le-Châtel, France

\begin{abstract}
We have developed an elastic viscoplastic model for beryllium, which can be easily incorporated into a computer code. The form of the equation is next to steinberg, Cochran and Guinan's model. The influence of temperature and strain rate is taken into account in the expressions of the elastic limit and the stress hardening function. The equation is settled from quasi static and dynamic compression tests. It can be then extrapolated over a wide range of stress, strain, strain rate and temperature. This allows to successfully calculate impacted sample profiles, as well as shock wave profiles.
\end{abstract}

\section{I./ IKTRODUCTION}

La demande de plus en plus importante de codes de calculs restituant la réponse de structures à des sollicitations dynamiques, nécessite le développement de modèles de comportement très elaborés. En effet, la résolution de ce type de problème fait appel, d'une part aux équations d'état et, d'autre part aux lois de comportement mécanique des matériaux en cause. La formulation des lois de comportement mécanique doit répondre à deux impératifs : retranscrire un ensemble de faits expérimentaux, et permettre de prédire le comportement du materiau dans des conditions rencontrees dans certaines experiences.

Initialement, les matériaux étaient considérés comme élastiques parfaitement plastiques. Une des premières améliorations a consisté à prendre en compte l'évolution de la limite d'élasticité en fonction de la vitesse de déformation et de la température. Dans le début des années 80, steinberg Cochran et Guinan /1/, ont proposé un nouveau modèle (dit S.C.G.), exprimant explicitement I'écrouissage. Celui-ci est aujourd'hui largement implante dans les codes de calculs. 
A partir de la formulation initiale de steinberg, nous avons développé un modèle qui retranscrit l'évolution de l'écrouissage en fonction de la vitesse de déformation et de la temperature. Le propos de cet article est done de démontrer les insuffisances du formalisme proposé par steinberg, et les améliorations que I'on peut apporter, illustrées au travers de l'étude d'un matériau : le béryllium.

\section{II. / WATERIAU}

Le matériau étudié est une nuance de béryllium S $200 \mathrm{~F}$, provenant d'un lingot 6laboré par BRUSH-WELLMAN. La taille moyenne des grains est de $15 \mu \mathrm{m}$. Le tableau suivant présente la composition chimique, en poids :

\begin{tabular}{ccccccccc}
\hline elément & Be & BeO & Fe & $C$ & $A 1$ & Mg & Si & autres \\
poids 8 & 99,1 & 0,9 & 0,08 & 0,09 & 0,03 & 0,01 & 0,02 & $<0.04$ \\
\hline
\end{tabular}

\section{III./ IOI DE COMPORTEIIENT}

\section{III.1/ Forme genérale}

Le modele S.C.G. traduit le comportement des matériaux sous la forme :

$Y=Y_{0}(1+\beta \cdot \varepsilon)^{n} \cdot(a+b \cdot T+g(P))$,

où $Y$ est la contrainte d'écoulement, $Y_{0}$ la limite élastique, $\varepsilon$ la déformation plastique, $\mathrm{T}$ la température, $\mathrm{P}$ la pression et $\boldsymbol{\beta}$ et $\mathrm{n}$ les paramètres $\alpha^{\prime}$ écrouissage, fonctions du matériau. Le module de cisaillement s'écrit :

$$
G=G_{0} \cdot(a+b \cdot T+g(P)) \text {. }
$$

Le formalisme propose par steinberg, Cochran et Guinan /1/ restitue correctement une courbe donnant la contrainte d'ecoulement en fonction de la deformation, pour une vitesse de déformation, $\dot{\varepsilon}$, et une température données. Mais ce modèle ne fait pas apparâtre $\dot{\varepsilon}$ alors qu'initialement, il était écrit pour prédire le comportement d'un matériau sous choc. De plus, I'influence de la température sur la contrainte d'écoulement, est prise linéaire. STEINBERG et LUND $/ 2 /$ ont récemment complété ce modele de façon à étendre sa validité dans un large domaine de vitesses de deformation.

Cette modification se manifeste dans la formulation de la contrainte d'écoulement $Y$. Ils remplacent la fonction $d^{\prime}$ ecrouissage $Y_{0} \cdot(1+\beta . \varepsilon)^{n}$ par la somme de deux termes :

$$
Y_{A} \cdot(1+\beta . \varepsilon)^{n}+Y_{T}(\dot{\varepsilon}, T)
$$

$Y_{A}$ est la composante athermique de la contrainte alors que $Y_{T}$, composante thermiquement activée, est fonction de la vitesse de déformation et de la température. 
La formulation de $Y_{T}$ nécessite l'introduction d'un certain nombre de nouveaux paramètres qui trouvent leur origine dans la description des mécanismes microscopiques avec, par exemple, I'utilisation de la notion de densité de dislocations ou du vecteur de Burgers. La justification physique de ce modèle est critiquable sur de nombreux points.

Ainsi, D.J.Steinberg et C.M. Lund font intervenir la notion de frottements visqueux dans l'évolution de la limite d'élasticité d’un matériau. Or, J.P.Ansart et R.Dormeval /3/ ont montré que celle ci ne peut être qu'une fonction thermiquement activée. C'est pourquoi, nous proposons d'écrire l'évolution du coefficient $Y_{0}$ sous la forme suivante :

$\mathrm{Y}_{0}=\Psi_{0}+\mathrm{k} \cdot \log \dot{\boldsymbol{\varepsilon}}$.

Par ailleurs, l'experience montre que, lorsque la vitesse de déformation crôt. I'écrouissage. $\left(Y /_{Y_{0}}\right)_{\varepsilon, T, P}$. devient plus important. Les valeurs de $\beta$ et $n$, paramètres $d^{\prime} 6$ crouissage, ne sont donc pas constantes, et sont des fonctions de $\dot{\varepsilon}$.

Enfin, pour une vitesse de déformation et une déformation fixées, nous constatons expérimentalement que la contrainte d'écoulement n'est généralement pas une fonction linéaire de la température. Nous avons donc choisi d'intégrer la temperature dans la variation des paramètres $\Psi_{0}, k$, ainsi que dans celle de $\beta$ et $n$.

En résumé, les paramètres $Y_{0}, \beta$ et $n$ de $I^{\prime}$ équation de Steinberg ne sont plus constants et deviennent des fonctions de la vitesse de deformation et de la température. Notre loi de comportement aura donc la forme globale suivante:

$$
\begin{aligned}
& Y=\left(\Psi_{0}(T)+k(T) \cdot \log \dot{\varepsilon}\right) \cdot(1+\beta(T, \dot{\varepsilon}) . \varepsilon)^{n(T, \dot{\varepsilon})}(1+g(P)) \\
& g(P) \text { donné par Steinberg }
\end{aligned}
$$

L'influence de la pression n'entrant pas dans le cadre de cette étude, nous avons conservé le formalisme du modèle s.c.G.

\section{III.2/ DBMARCHE BXPERIMENTALE.}

Nous avons réalisé des essais de compression sous sollicitations quasi statiques et dynamiques sur des échantillons de béryllium $\$ 200 \mathrm{~F}=8 \mathrm{~mm}, \mathrm{~h}=8 \mathrm{~mm}$. Nous avons atteint cinq vitesses de déformation: $2.10^{-4}, 2.10^{-2}, 2, * 500$ et $\approx 750 \mathrm{~s}^{-1}$. A chaque vitesse, 1 'échantilion a été porté à quatre températures différentes: 20 , 100,200 et $300^{\circ} \mathrm{C}$. Chaque expérience fournit ainsi une courbe de la contrainte d'écoulement en fonction de la déformation. Certaines d'entre elles sont présentées figures 1 à 3 . Chacune est modélisée par une équation du type:

$$
Y=Y_{0}(1+\beta . \varepsilon)^{n} \text {. }
$$

Nous étudions ensuite I'influence de la vitesse de déformation sur les paramètres $Y_{0}, \beta$ et $n$, puis leur évolution en fonction de la température. Nous obtenons ainsi la loi de comportement du béryllium S200F: 
$Y=\left(\Psi_{0}(T)+k(T) \cdot \log \dot{\varepsilon}\right) \cdot(1+\beta(T, \dot{\varepsilon}) \cdot \varepsilon)^{n(T, \dot{\varepsilon})}$ avec

$\Psi_{0}(\mathrm{~T})=282,3-0,229 . \mathrm{T} \mathrm{MPa}$ et $\mathrm{k}(\mathrm{T})=16,76 \mathrm{MPa}$

$R(\dot{\varepsilon}, T)=\left(194,3 . e^{T \cdot 7,12.10^{-3}}\right) \cdot \dot{\varepsilon}^{-0,1412 . e^{-T \cdot 1,905.10^{-3}}}$

$n(\dot{\varepsilon}, T)=-0,02559+\frac{111}{T+273}+\left(2,282 \cdot 10^{-2} \cdot e^{-T \cdot 4,42 \cdot 10^{-3}}\right) \cdot \log \dot{\varepsilon}+\left(2,12 \cdot 10^{-4}+\frac{0,117}{T+30}\right) \cdot \dot{\varepsilon}^{0,45}$

$g(P)=15,5 \cdot 10^{-12} \cdot P \cdot \frac{p}{\rho_{0}}$ où $P$ est la pression en $\mathrm{Pa}$,

$p_{0}=1850 \mathrm{~kg} \cdot \mathrm{m}^{-3}$ et $p$ la masse volumique en $\mathrm{kg} \cdot \mathrm{m}^{-3}$.
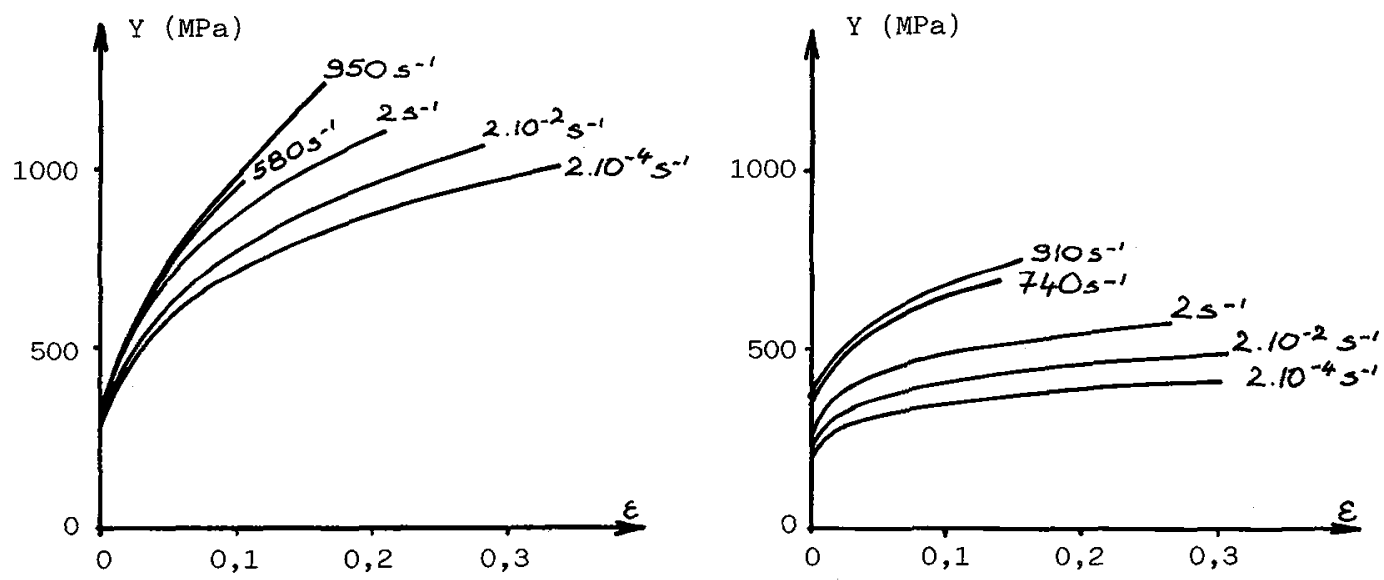

Figure 1: Contrainte d'écoulement à $20^{\circ} \mathrm{C}$ Figure 2: Contrainte d'écoulement à $300^{\circ} \mathrm{C}$

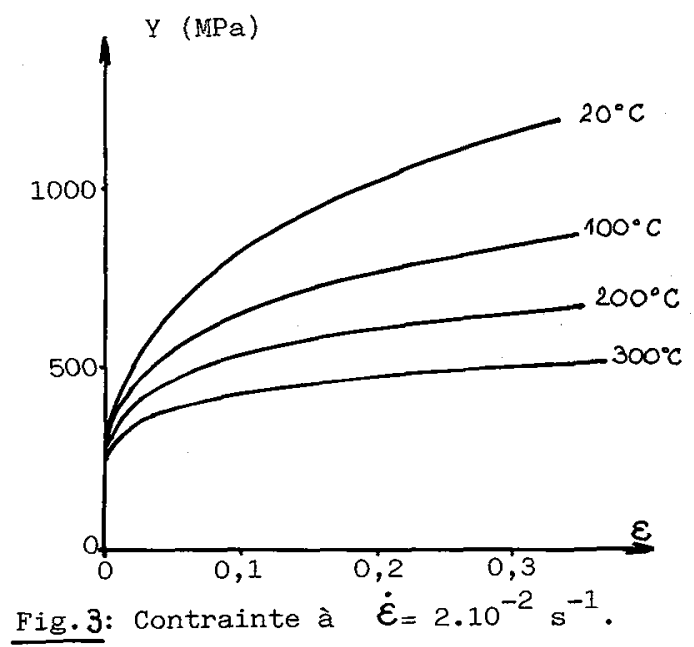

La formule finale prend une allure complexe. Le choix d'écrire un modele valable sur une large plage de deformations, vitesses de déformation et temperatures 
en est la conséquence directe. Si l'on cherche à restreindre ce domaine, la formule se simplifie, certaines expressions devenant quasiment constantes.

Cette Ioi est introduite dans un code de calcul, afin de pouvoir I'extrapoler pour des deformations et des vitesses de déformation plus importantes. Comme nous allons I'exposer, les essais de Taylor et de choc de plaques ont permis la confrontation entre l'expérience et les calculs.

\section{IV/ CALCULS}

\section{IV.1/ Conditions des calculs}

L'équation d'état utilisee est une equation de type Mie-Grüneisen : $P=C \mu+D \mu^{2}+S \mu^{3}+\Gamma \rho E$.

avec $\mu=\rho_{/} \rho_{0} \cdot 1$, déformation,

$\rho_{0}$, densité initiale,

$\mathrm{E}$, ênergie interne,

$\Gamma$, coefficient de Grüneisen,

C, mođule d'élasticité,

$\mathrm{D}$ et $\mathrm{S}$, constantes du matériau.

Différentes lois de comportement ont été utilisées :

- une loi traduisant un comportement élastique parfaitement plastique $\left(Y_{0}=330 \mathrm{MPa}\right)$. - la formule originale de steinberg, $\mathrm{Y}=330 .(1+\mathrm{f}(\mathrm{T})+\mathrm{g}(\mathrm{P})) \cdot(1+26 . \varepsilon)^{0,78} \mathrm{MPa}$, - notre modèle (cf. ci-dessus).

Les paramètres numériques (maillage, conditions aux limites..) sont restés constants. Le code, couplé Euler-Lagrange, possède un schéma eulérien d'ordre deux, et permet l'introduction de lignes de glissement aux interfaces Euler-Lagrange ou Lagrange-Lagrange.

\section{IV.2/ Bssais de Taylor}

Des échantillons de beryllium s200F, $\phi=8 \mathrm{~mm}, \mathrm{~h}=20 \mathrm{~mm}$, ont subi un impact sur une cible rigide, à différentes vitesses d'impact et différentes températures $\left(150 \mathrm{~m} / \mathrm{s}<\mathrm{v}_{\text {impact }}<250 \mathrm{~m} / \mathrm{s}, 20^{\circ} \mathrm{C}<\mathrm{T}<300^{\circ} \mathrm{C}\right)$. Les profils finals des échantillons sont relevés. En parallele, le code de calcul, dans lequel nous avons introduit notre modèle, effectue des prévisions, en partant des conditions initiales correspondantes à ces expériences. Ia figure 4 compare les profils expérimentaux, ceux calculés avec notre loi de comportement et ceux calculés avec la loi de comportement proposée par steinberg $/ 1 /$, pour $V_{\text {impact }}=216 \mathrm{~m} / \mathrm{s}$ et $\mathrm{T}=20^{\circ} \mathrm{C}$ et $\mathrm{T}=300^{\circ} \mathrm{C}$.

A $20^{\circ} \mathrm{C}$, pour une vitesse de tir de $216 \mathrm{~m} / \mathrm{s}$, notre modèle, aussi bien que celui de steinberg, rend compte du comportement du béryllium de façon correcte. I'écart n'excède pas, en effet 3\%. Cependant, notre modelle améliore la restitution. En 
effet, le profil final est plus conforme à l'expérience dans notre cas, surtout dans la zone la plus deformée, plus proche de l'impact.
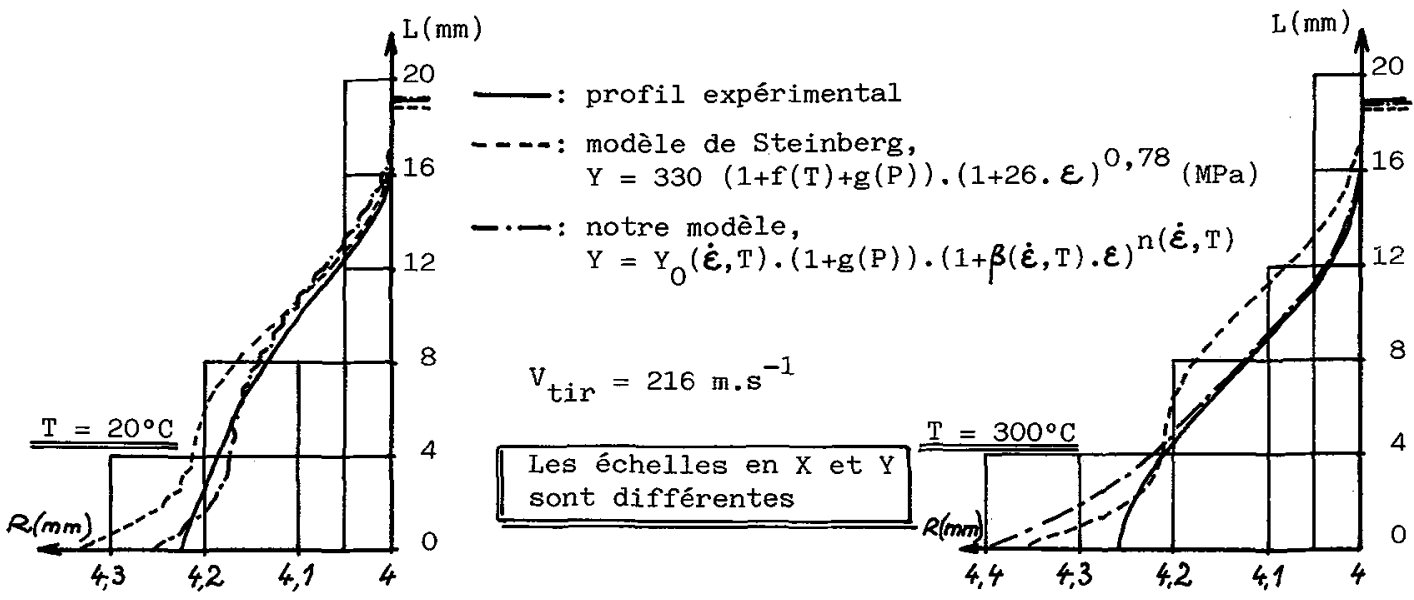

Figure 4: Essai de Taylor. Comparaison du profil expérimental et des calculs.

A $300^{\circ} \mathrm{C}$, les deux modèles, là encore, restituent correctement l'expérience, puisque l'erreur est inférieure à 5\%. Pourtant le tracé obtenu à partir de l'expression de Steinberg fait apparaftre un point d'inflexion à $L * 4 \mathrm{~mm}$, qui n'existe pas sur le profil experimental, et qui peut introduire localement des concentrations artificielles de contraintes dans les calculs. Par contre, notre modèle est plus proche de la forme globale de l'échantillon. Entre $L=18,9 \mathrm{~mm}$ et $\mathrm{L}=4 \mathrm{~mm}$, le tracé expérimental et celui de notre formulation sont à peine distincts. L'écart observé entre $I=4 \mathrm{~mm}$ et $L=0 \mathrm{~mm}$ est probablement da a un frottement que nous ne sommes pas en mesure d'apprécier. L'absence de point d'inflexion, dans notre cas, nous laisse penser que les calculs resteront fideles aux experiences sur une plage de déformations plus importante qu'avec le modèle s.C.G..

Nous avons aússi calculé la vitesse de déformation, dans quelques mailles au cours du temps. Les résultats, non présentés ici, confirment que ces grandeurs dépassent, localement, $10^{4} \mathrm{~s}^{-1}$. Notre modèle a donc été globalement extrapolé en vitesse de déformation. En revanche, le caractère peu ductile du beryllium ne nous a pas permis d'obtenir de déformations supérieures à 0,2 , même localement.

\section{IV.3/ Choc de plaques}

Des échantilions de béryllium $\$ 200 \mathrm{~F}, \emptyset=30 \mathrm{~mm}, \mathrm{~h}=4 \mathrm{~mm}$, ont subi un essai 
de choc de plaques. Nous présentons ci dessous quelques conditions d'essais retenues :

\begin{tabular}{|c|c|c|}
\hline Impacteur & Vit. de tir (m/s) & Pression de choc (GPa) \\
\hline AU4G & 112 & 0,97 \\
AU4G & 197 & 1,63 \\
AU4G & 464 & 3,73 \\
Tantale & 485 & 6,12 \\
\hline
\end{tabular}

AU4G : alliage d'aluminium (2017 norme americaine)

Un système d'Interférometrie Doppler Laser a permis d'enregistrer l'évolution de la vitesse de surface libre des échantillons au cours du temps. Les résultats expérimentaux sont comparés aux calculs effectués à partir de notre loi de comportement et de celle de steinberg (figure 5). Sur les graphes ci-dessous, nous avons ajouté un profil theorique, obtenu a partir d'une loi elastique parfaitement plastique, en omettant 1 'interaction entre le précurseur élastique et l'onde plastique.
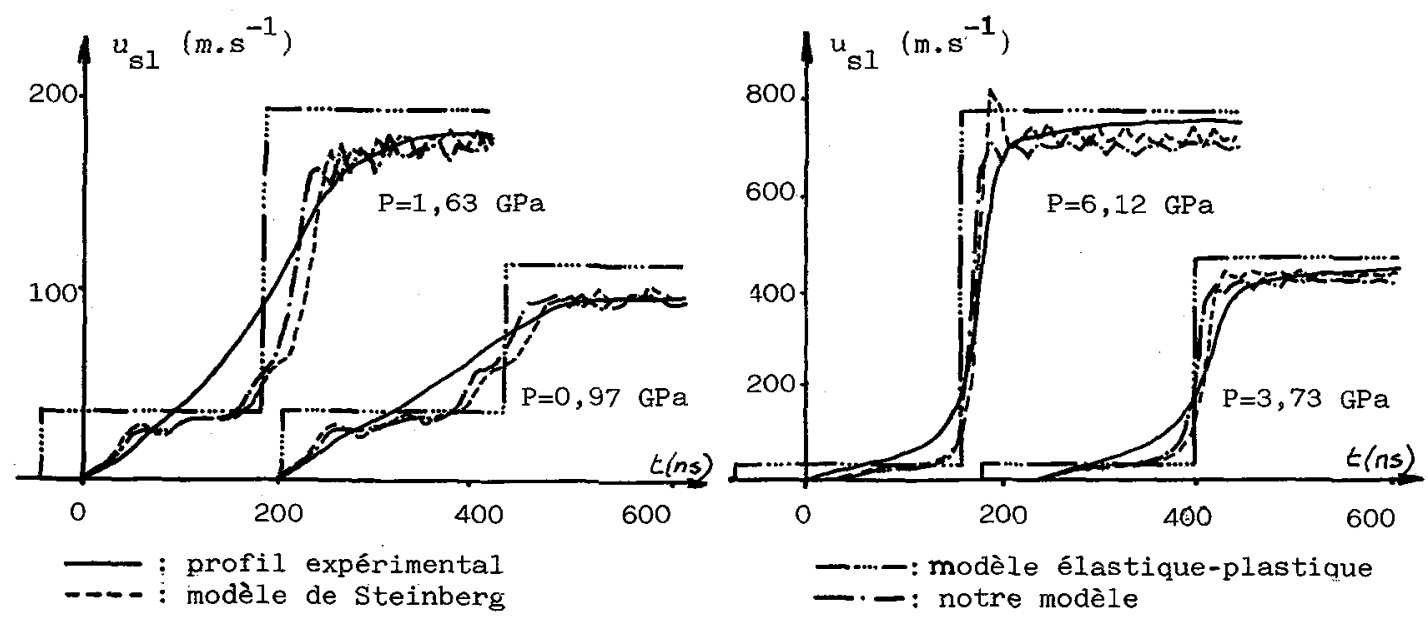

Figure 5: Essais de choc de plaques. $T=20^{\circ} \mathrm{C}$. Comparaison entre expérience et calculs

La loi S.C.G. et la nótre montrent un accord acceptable entre l'expérience et les calculs. Cette faible distinction entre les deux expressions paraft d'autant plus normale, que les expériences de choc rentrent dans le cadre initial de validité du modele S.C.G..

Cependant, les fronts de montée ne sont pas restitues très fidèlement, dans aucun des cas. Le domaine de pressions de choc explore par steinberg pour valider son modèle est largement plus blevé que le nôtre; dans ce cas l'accord entre l'expérience et le modèle S.C.G. est meilleur $/ 5 /$. 
Par ailleurs, les deux courbes calculées donnent une bonne restitution du temps de mise en vitesse. De même, le niveau maximum de vitesse atteint est évalué de facon correcte (erreur relative inférieure à 5\%), par rapport à l'experience.

Les essais de choc de plaques donnent ainsi une extrapolation correcte de notre loi de comportement, à des vitesses de déformation voisine de $10^{5} \mathrm{~s}^{-1}$. Une autre solution consiste à étudier la mise en vitesse d'une interface béryllium-fluorure de lithium (LiF). Dans ce cas, on $s^{\prime}$ affranchit des phénomènes d'interaction entre le précurseur elastique et 1 'onde plastique.

\section{vI./ CONCLUSION}

Nous avons mis au point un modele elasto visco plastique, utilisable par un code de calcul. Par rapport au modèle steinberg, Cochran et Guinan, la principale innovation est la prise en compte de l'influence de la température et de la vitesse de déformation dans l'évolution de la limite d'élasticité et de l'écrouissage. En effet, l'expérience montre que l'on ne peut pas considérer la fonction d'écrouissage indépendante de $\dot{\varepsilon}$ et de T. A partir de ces observations expérimentales, et de quelques considerations physiques, nous avons écrit un modele qui retranscrit les points expérimentaux.

Cette loi de comportement, a été établie pour du béryllium \$200F, à partir des essais de compression quasi statiques et dynamiques, pour des déformations inférieures à 0,15 et des vitesses de déformation qui varient entre $2.10^{-4}$ et $\approx 10^{3} \mathrm{~s}^{-1}$. Les essais d'impact ont induit des deformations et des vitesses de déformation locales respectivement de 0,2 et de $10^{4} \mathrm{~s}^{-1}$. Avec les essais de chocs de plaques, nous avons atteint des vitesses de déformation de $10^{4}$ à $10^{6} \mathrm{~s}^{-1}$. En introduisant notre modèle dans un code de calcul, nous avons pu extrapoler notre loi de comportement par comparaison entre l'expérience et les prévisions. Notre formule est ainsi valable sur une large gamme de deformations, de vitesses de deformation, et de temperatures.

$11 /$ : A constitutive model for metals, applicable at high strain rate. D.J.STEINBERG, S.G.COCHRAN, M.W.GUTNAN. J.Appl.PhYs. Vol 51 (3) . p1498. 1980

$12 /$ : A constitutive model for strain rates from $10^{-4}$ to $10^{6} \mathrm{~s}^{-1}$. STEINBERG D.J., LUND C.M. (1989) J. Appl. Phys. 65.p.1528.

/3/ : About the strain rate dependence of yield stress and flow stress of materials. 2nd symp. on plasticity and its current applications.

J.P.ANSART, R.DORMEVAL. Pergamon Press. p 371. Tsu, Japan. 1989

14/ : Steady wave rise time and spall measurement on uranium (3-15 GPa) . D.E.GRADY. In "Metallurgical applications of shock wave and high strain rate phenomena". 1986. p 763. Ed. by MURR, STAUDHAMMER, MEYERS.

15/ : The effect on work-hardening in dynamic deformation of beryllium. D.J.STEINBERG, L.L.N.L, Livermore, CA. UCID 20243 (1985) 